\title{
A Generalized ML-Hyers-Ulam Stability of Quadratic Fractional Integral Equation
}

https://doi.org/10.1515/nleng-2021-0033

Received Aug 15, 2021; accepted Oct 7, 2021.

\begin{abstract}
An interesting quadratic fractional integral equation is investigated in this work via a generalized Mittag-Leffler (ML) function. The generalized ML-HyersUlam stability is established in this investigation. We study both of the Hyers-Ulam stability (HUS) and MLHyers-Ulam-Rassias stability (ML-HURS) in detail for our proposed differential equation (DEq). Our proposed technique unifies various differential equations' classes. Therefore, this technique can be further applied in future research works with applications to science and engineering.
\end{abstract}

Keywords: Stability; quadratic fractional integral equation; Mittag-Leffler function

MSC: 45M10, 65P40, 65L20, 70H14

\section{Introduction}

Several types of integral equations (IEs) are considered very important in various functional analysis topics because of their essential role in engineering, physics, economics, and natural sciences. Many real-life applications can be well described using a suitable quadratic fractional integral equation. For instance, quadratic integral

\footnotetext{
*Corresponding Author: Mohammed K. A. Kaabar, Jabalia Camp, United Nations Relief and Works Agency (UNRWA), Palestinian Refugee Camp, Gaza Strip Jabalya, State of Palestine Institute of Mathematical Sciences, Faculty of Science, University of Malaya, Kuala Lumpur 50603, Malaysia,

E-mail:mohammed.kaabar@wsu.edu

Vida Kalvandi, Department of Mathematics, Razi University, Kermanshah, Iran

Nasrin Eghbali, Department of Mathematics, Faculty of Sciences, Mohaghegh Ardabili, Ardabil, Iran

Mohammad Esmael Samei, Department of Mathematics, Faculty of Basic Science, Bu-Ali Sina University, Hamedan, Iran

Zailan Siri, Institute of Mathematical Sciences, Faculty of Science, University of Malaya, Kuala Lumpur 50603, Malaysia

Francisco Martínez, Department of Applied Mathematics and Statistics, Technological University of Cartagena, Cartagena 30203, Spain
}

equations (QIEs) play an important role in kinetic molecular, radiative, neutron transport, traffic, and queuing theories $[1,2]$.

The fundamental idea behind the theory of functional equations' stability was first initiated in 1940 when Ulam studied the group homomorphism problem's stability in [3]. Then, in the following year, that problem was investigated by Hyers for the Banach spaces (BaSps) case. As a result, this particular stability is named as HUS. A generalized form of the Hyers' theorem was studied by Rassias [4] in 1978 where the stability was investigated via unbounded Cauchy differences. Thus, this contribution is named as HURS. By interchanging the functional equation with an inequality that acts as an initial equation's perturbation, the functional equation's stability problem has been given a special priority in research studies [5]. The HUS of DEq with an integer order was first studied by Alsina and Ger [6]. An extension of analysis to fractional differential equations was investigated in many research works [7, 8]. Various ML-Hyers-Ulam stability's types have been proposed in [9] for the fractional integral equation (FIE), and its exact solution can be approximated from every FIE's mapping.

Fractional calculus (FC) constitutes an essential tool for analysis when the arbitrary-order integrals and derivatives are investigated via the generalizations of integerorder differentiation and $\mathrm{n}$-fold integration [10-14]. Some interesting research works concerning the relation between symmetry and generalized fractional calculus properties have been conducted [15]. Although studying FC was focused on the pure mathematical investigation, various natural sciences and technology fields have studied them such as mathematical physics, fluid mechanics, image processing, biology, and entropy which reveal the powerful applicability of FC in modeling scientific phenomena [16-24]. Theoretically and practically, FC has received a global research interest $[25,26]$. There are equivalent types of differential equations to quadratic fractional integral equation which acquire soliton solutions to have more effective model equation than other related ones. 
A notable approximation type is Padé-type approximation with lump solutions (see [27]). The $N$-soliton solutions to integrable equations have been recently investigated via the Hirota bilinear method for both $(1+1)$-dimensional integrable equations [28] and ( $2+1)$-dimensional integrable equations [29] (see also [30-32] for examples). For more background information and examples of soliton solutions, we refer to [33-35]. While QIEs have several applications in natural sciences and engineering, investigating QIEs with the help of fractional calculus can provide us with a very powerful tool in modeling phenomena in science and engineering, particularly queuing theory and biology [36] (see also [37-39]), due to the importance of nonlocality and memory effect of the proposed fractional models when we discuss the systems' dynamics and fractional behavior. Some numerical approaches for the ML distribution have been studied such as Monte Carlo approach for a random number generator for the one-parameter ML distribution [40], Grünwald-Letnikov approach in the sense of equivalent fractional integrator [41], rational approximation for the two-parameter ML function with discrete elliptic operator [42]. In addition, multi-parameter extensions of the ML distribution have been recently studied in [43].

This work investigates the following quadratic FIE’s stability:

$$
y(t)=\mathcal{V}(t, y(t))\left[\frac{1}{\Gamma(q)} \int_{0}^{t}(t-\xi)^{q-1} Q_{\alpha, \beta, \delta}^{\gamma, q, r}\left((t-\xi)^{q}\right) \mathcal{W}(\xi, y(\xi)) \mathrm{d} \xi\right],
$$

where $\mathcal{V}, \mathcal{W}: J \times \mathbf{R} \rightarrow \mathbf{R}$ are continuous functions (CFs), $q \in[1,2), \Gamma$ represents Gamma function(GF), and $Q_{\alpha, \beta, \delta}^{\gamma, q, r}$ is the generalized ML function. The quadratic operator equations' existence can be shown under the conditions of mixed Lipschitz and compactness along with a certain growth condition on the nonlinearities included in the quadratic operator.

This work is outlined as follows: some essential notions about generalized metric and ML function are discussed in Sec. 2. Our main results are obtained in both Sec. 3 and Sec. 4. Our work is concluded in Sec. 5.

\section{Essential Concepts}

For a nonempty set $y$, the generalized metric on $y$ is introduced in this section. Given a function: $\hat{\rho}: y \times y \rightarrow[0,+\infty]$, named as a generalized metric on $y$ iff the assumptions below are satisfied:

(A1) $\hat{\rho}\left(y_{1}, y_{2}\right)=0$ iff $y_{1}=y_{2}$.

(A2) $\hat{\rho}\left(y_{1}, y_{2}\right)=\hat{\rho}\left(y_{2}, y_{1}\right) \forall y_{1}, y_{2} \in \mathcal{y}$.

(A3) $\hat{\rho}\left(y_{1}, y_{2}\right) \leq \hat{\rho}\left(y_{1}, y_{3}\right)+\hat{\rho}\left(y_{3}, y_{2}\right) \forall y_{i} \in y$ with $i=1,2,3$.

It is obvious that the above definition is different from the usual complete metric space definition where not every two points in $y$ have necessarily a finite distance. Therefore, this space can be named as a generalized complete metric space (GCMSp).

Banach's fixed point theorem (BFPThm) in a GCMSp is expressed as:

Theorem 2.1. Suppose that $(y, \hat{\rho})$ is a GCMSp. Let us assume that $\mathcal{O}: y \rightarrow y$ is a strictly contractive operator with the Lipschitz constant $\ell<1$. If $\exists$ a nonnegative integer $k \ni$

$$
\hat{\rho}\left(\mathcal{O}^{k+1}(y), \mathcal{O}^{k}(y)\right)<\infty,
$$

for some $y \in y$, then the following are true:

(I) The sequence $\mathcal{O}^{n}(y)$ converges to a fixed point $y^{\star}$ of $\mathcal{O}$.

(II) $y^{\star}$ is the unique fixed point of $\mathcal{O}$ in

$$
y^{*}=\left\{y \in y \mid \hat{\rho}\left(\mathcal{O}^{k}\left(y^{\star}\right), y\right)<\infty\right\} .
$$

(III) If $y \in y^{\star}$, then we have:

$$
\hat{\rho}\left(y, y^{\star}\right) \leq \frac{1}{1-\ell} \hat{\rho}(\mathcal{O}(y), y) .
$$


Definition 2.2. [44] (ML function) The one-parameter $M L$ function, denoted by $\mathfrak{E}_{\alpha}(z)$, is written as:

$$
\mathcal{E}_{\alpha}(z)=\sum_{k=0}^{\infty} \frac{1}{\Gamma(1+\alpha k)} z^{k},
$$

where $z, \alpha \in \mathbf{C}, \operatorname{Re}(\alpha)>0$. If we substitute $\alpha=1$ in the above equation, then we have:

$$
\mathcal{E}_{1}(z)=\sum_{k=0}^{\infty} \frac{z^{k}}{\Gamma(1+k)}=\sum_{k=0}^{\infty} \frac{z^{k}}{k !}=e^{z} .
$$

Definition 2.3. In 1905, Wiman proposed the generalized form of $\mathcal{E}_{\alpha}(z)$ [45]. Then, both Agarwal [46], and Humbert and Agarwal [47] introduced a function as follows:

$$
\varepsilon_{\alpha, \beta}(z)=\sum_{k=0}^{\infty} \frac{1}{\Gamma(\beta+\alpha k)} z^{k}
$$

where $z, \alpha, \beta \in \mathbf{C}, \operatorname{Re}(\alpha)>0, \operatorname{Re}(\beta)>0$.

Prabhakar generalized in 1971 this function in the following form:

$$
\mathcal{E}_{\alpha, \beta}^{\gamma}(z)=\sum_{k=0}^{\infty} \frac{(\gamma)_{k}}{\Gamma(\beta+\alpha k)} z^{k}
$$

where $z, \alpha, \beta, \gamma \in \mathbf{C}, \operatorname{Re}(\alpha)>0, \operatorname{Re}(\beta)>0, \operatorname{Re}(\gamma)>0$, such that $\gamma \neq 0$,

$$
(\gamma)_{k}=\prod_{i=0}^{k-1}(\gamma+i),
$$

which is called the Pochhammer symbol [48] $\ni(\gamma)_{k}=\frac{\Gamma(\gamma+k)}{\Gamma(\gamma)}$. Another generalization of this function was introduced in 2007 by Shulka and Prajapati in [48] as:

$$
\mathcal{E}_{\alpha, \beta}^{\gamma, q}(z)=\sum_{k=0}^{\infty} \frac{(\gamma)_{q k}}{k ! \Gamma(\beta+\alpha k)} z^{k}
$$

where $z, \alpha, \beta, \gamma \in \mathbf{C}$,

$$
\min \{\operatorname{Re}(\alpha), \operatorname{Re}(\beta), \operatorname{Re}(\gamma)\}>0,
$$

and $q \in(0,1) \cup \mathbf{N}$. In 2009, again Shalka and Prajapati [49] introduced a generalized ML function. In 2012, a novel generalized form of ML function was proposed by both Salim in [50], and by Amit and Saraswat in [51] as:

$$
\varepsilon_{\alpha, \beta, \delta}^{\gamma, q}(z)=\sum_{k=0}^{\infty} \frac{(\gamma)_{q k}}{(\delta)_{q k} \Gamma(\beta+\alpha k)} z^{k}
$$

where $z, \alpha, \beta, \gamma \in \mathbf{C}$, Eq. (2.5) holds, $q \in(0,1) \cup \mathbf{N}$ and

$$
(\gamma)_{q k}=\frac{\Gamma(\gamma+q k)}{\Gamma(\gamma)}, \quad(\delta)_{q k}=\frac{\Gamma(\delta+q k)}{\Gamma(\delta)},
$$

denote the generalized Pochhammer symbol [48]. After them, in 2016 Desal et al. [52] introduced another definition of generalized ML function.

Definition 2.4. [53]. The generalized ML function, denoted by $\mathcal{Q}_{\alpha, \beta, \delta}^{\gamma, q, r}(y)$, can be expressed as:

$$
\begin{aligned}
\mathcal{Q}_{\alpha, \beta, \delta}^{\gamma, q, r}(z) & =\mathcal{Q}_{\alpha, \beta, \delta}^{\gamma, q, r}\left(a_{1}, a_{2}, \ldots, a_{r}, b_{1}, b_{2}, \ldots, b_{r}, z\right) \\
& =\sum_{s=0}^{\infty} \frac{\prod_{n=1}^{r} \beta\left(b_{n}, s\right)(\gamma)_{q s}}{\prod_{n=1}^{r} \beta\left(b_{n}, s\right)(\delta)_{q s} \Gamma(\beta+\alpha s)} z^{s},
\end{aligned}
$$

where $y, \alpha, \beta, \gamma, \delta, a_{i}, b_{i} \in \mathbf{C}$, Equation (2.5) holds, $q \in(0,1) \cup \mathbf{N},(\gamma)_{q k}$ and $(\delta)_{q k}$ are defined in (2.7). 


\section{Hyers-Ulam-Rassias Stability}

The HURS and HUS of equation (1.1) are investigated in this section on a compact interval $[0, a]$.

Definition 3.1. If for each given function y satisfies

$$
\left|y(t)-\mathcal{V}(t, y(t))\left[\frac{1}{\Gamma(q)} \int_{0}^{t}(t-\xi)^{q-1} \mathcal{Q}_{\alpha, \beta, \delta}^{\gamma, q, r}\left((t-\xi)^{q}\right) \mathcal{W}(\xi, y(\xi)) \mathrm{d} \xi\right]\right| \leq \varepsilon \varphi(t),
$$

$\exists$ an equation's (1.1) solution $u_{0}$ and a constant, $c>0$, which is independent of both $y$ and $u_{0} \ni$

$$
\left|y(t)-u_{0}(t)\right| \leq c \varepsilon \varphi(t),
$$

for $t \in[a, b]$, then equation (1.1) is named as Hyers-Ulam-Rassias stable. On the other hand, when $\varphi$ is formed as a constant function, equation (1.1) is named as Hyers-Ulam stable.

Theorem 3.2. For a closed and bounded interval $J=[0, a]$ of the real line $\mathbf{R}$ for some $a>0$, suppose that $\mathcal{V}$ and $\mathcal{W}$ : $J \times \mathbf{R} \rightarrow \mathbf{R}$ are CFs, $q \in[1,2)$ and a gamma function, denoted by $\Gamma$, the following are satisfied:

$$
|\mathcal{V}(t, y(t))-\mathcal{V}(t, u(t))| \leq M_{v}|y(t)-u(t)|
$$

and

$$
|\mathcal{W}(t, y(t))-\mathcal{W}(t, u(t))| \leq M_{w}|y(t)-u(t)|,
$$

for each $t \in J, y, u \in \mathbf{R}$, and suppose that

$$
\left|y(t)-\mathcal{V}(t, y(t))\left[\frac{1}{\Gamma(q)} \int_{0}^{t}(t-\xi)^{q-1} \mathcal{Q}_{\alpha, \beta, \delta}^{\gamma, q, r}\left((t-\xi)^{q}\right) g(\xi, y(\xi)) \mathrm{d} \xi\right]\right| \leq \varepsilon,
$$

and we also suppose that

$$
\begin{aligned}
0 & <\left(M_{v} M_{w} K \varepsilon+M_{v}\|\mathcal{W}\|+M_{w}\|\mathcal{V}\|\right)\left[\frac{1}{\Gamma(q)} \sum_{m=0}^{\infty} \frac{\prod_{n=1}^{r} \beta\left(b_{n}, m\right)(\gamma)_{q m}}{\prod_{n=1}^{r} \beta\left(a_{n}, m\right)(\delta)_{q m} \Gamma(\beta+\alpha m)} \cdot\left(\frac{t^{q(m+1)}}{q(m+1)}\right)\right] \\
& =K^{\prime}<1 .
\end{aligned}
$$

Then, the quadratic FIE is Hyers-Ulam stable.

Proof. Let us consider the continuous functions space: $y=C([0, a], \mathbf{R})$ with a generalized metric (GMr), expressed as:

$$
\hat{\rho}(g, h)=\inf \{K \in[0, \infty]:|g(x)-h(x)| \leq K \varepsilon, \forall t \in J\} .
$$

By referring to the discussed notions in Sec. 2, it is very clear that $(y, \hat{\rho})$ is a GCMSp (see Theorem 2.1). Let us now construct an operator: $\mathcal{O}: y \rightarrow y$ as:

$$
\mathcal{O}(y(t))=\mathcal{V}(t, y(t))\left[\frac{1}{\Gamma(q)} \int_{0}^{t}(t-\xi)^{q-1} Q_{\alpha, \beta, \delta}^{\gamma, q, r}(t-\xi)^{q} \mathcal{W}(\xi, y(\xi)) \mathrm{d} \xi\right] .
$$

From the definition of $\mathcal{O}$ and equations (3.1) and (3.2), we obtain:

$$
\begin{aligned}
|\mathcal{O}(y(t))-\mathcal{O}(u(t))|= & \mid \mathcal{V}(t, y(t))\left[\frac{1}{\Gamma(q)} \int_{0}^{t}(t-\xi)^{q-1} Q_{\alpha, \beta, \delta}^{\gamma, q, r}(t-\xi)^{q} \mathcal{W}(\xi, y(\xi)) \mathrm{d} \xi\right] \\
& -\mathcal{V}(t, u(t))\left[\frac{1}{\Gamma(q)} \int_{0}^{t}(t-\xi)^{q-1} Q_{\alpha, \beta, \delta}^{\gamma, q, r}(t-\xi)^{q} \mathcal{W}(\xi, u(\xi)) \mathrm{d} \xi\right] \mid
\end{aligned}
$$




$$
\begin{aligned}
& \leq|\mathcal{V}(t, y(t))-\mathcal{V}(t, u(t))|\left[\frac{1}{\Gamma(q)} \int_{0}^{t}(t-\xi)^{q-1} Q_{\alpha, \beta, \delta}^{\gamma, q, r}(t-\xi)^{q}|\mathcal{W}(\xi, y(\xi))-\mathcal{W}(\xi, u(\xi))| \mathrm{d} \xi\right] \\
& +|\mathcal{V}(t, y(t))-\mathcal{V}(t, u(t))|\left[\frac{1}{\Gamma(q)} \int_{0}^{t}(t-\xi)^{q-1} Q_{\alpha, \beta, \delta}^{\gamma, q, r}(t-\xi)^{q}|\mathcal{W}(\xi, u(\xi))| \mathrm{d} \xi\right] \\
& +|\mathcal{V}(t, u(t))|\left[\frac{1}{\Gamma(q)} \int_{0}^{t}(t-\xi)^{q-1} Q_{\alpha, \beta, \delta}^{\gamma, q, r}(t-\xi)^{q}|\mathcal{W}(\xi, y(\xi))-\mathcal{W}(\xi, u(\xi))| \mathrm{d} \xi\right] \\
& \leq M_{v}|y(t)-u(t)|\left[\frac{1}{\Gamma(q)} \int_{0}^{t}(t-\xi)^{q-1} Q_{\alpha, \beta, \delta}^{\gamma, q, r}(t-\xi)^{q} M_{w}|y(\xi)-u(\xi)| \mathrm{d} \xi\right] \\
& +M_{v}|y(t)-u(t)|\left[\frac{1}{\Gamma(q)} \int_{0}^{t}(t-\xi)^{q-1} Q_{\alpha, \beta, \delta}^{\gamma, q, r}(t-\xi)^{q}\|\mathcal{W}\| \mathrm{d} \xi\right] \\
& +\|\mathcal{V}\|\left[\frac{1}{\Gamma(q)} \int_{0}^{t}(t-\xi)^{q-1} Q_{\alpha, \beta, \delta}^{\gamma, q, r}(t-\xi)^{q} M_{w}|y(\xi)-u(\xi)| \mathrm{d} \xi\right] \\
& \leq\left(M_{v} M_{W} K^{2} \varepsilon^{2}+M_{v} K \varepsilon\|\mathcal{W}\|+M_{v} K \varepsilon\|\mathcal{V}\|\right)\left[\frac{1}{\Gamma(q)} \int_{0}^{t}(t-\xi)^{q-1} Q_{\alpha, \beta, \delta}^{\gamma, q, r}(t-\xi)^{q} \mathrm{~d} \xi\right] \\
& \leq\left(M_{v} M_{w} K^{2} \varepsilon^{2}+M_{v} K \varepsilon\|\mathcal{W}\|+M_{w} K \varepsilon\|\mathcal{V}\|\right)\left[\frac{1}{\Gamma(q)} \int_{0}^{t}(t-\xi)^{q-1}\right. \\
& \left.\times \sum_{m=0}^{\infty} \frac{\prod_{n=1}^{r} \beta\left(b_{n}, m\right)(\gamma)_{q m}}{\prod_{n=1}^{r} \beta\left(a_{n}, m\right)(\delta)_{q m} \Gamma(\beta+\alpha m)}(t-\xi)^{m q} \mathrm{~d} \xi\right] \\
& =\left(M_{v} M_{w} K^{2} \varepsilon^{2}+M_{v} K \varepsilon\|\mathcal{W}\|+M_{w} K \varepsilon\|\mathcal{V}\|\right) \\
& \times\left[\frac{1}{\Gamma(q)} \sum_{m=0}^{\infty} \frac{\prod_{n=1}^{r} \beta\left(b_{n}, m\right)(\gamma)_{q m}}{\prod_{n=1}^{r} \beta\left(a_{n}, m\right)(\delta)_{q m} \Gamma(\beta+\alpha m)} \int_{0}^{t}(t-\xi)^{q-1}(t-\xi)^{m q} \mathrm{~d} \xi\right] \\
& \leq K \varepsilon\left(M_{v} M_{w}+M_{v}\|\mathcal{W}\|+M_{w}\|\mathcal{V}\|\right) \\
& \times\left[\frac{1}{\Gamma(q)} \sum_{m=0}^{\infty} \frac{\prod_{n=1}^{r} \beta\left(b_{n}, m\right)(\gamma)_{q m}}{\prod_{n=1}^{r} \beta\left(a_{n}, m\right)(\delta)_{q m} \Gamma(\beta+\alpha m)} \cdot\left(\frac{t^{q(m+1)}}{q(m+1)}\right)\right] .
\end{aligned}
$$

Because $0<K^{\prime}<1$, we conclude that $\mathcal{O}$ is contraction mapping. Let us take $y_{0}^{\prime} \in \mathcal{y}$, from the continuous property of $y_{0}^{\prime} \in \mathcal{Y}$ and $\mathcal{O}\left(y_{0}^{\prime}\right) \in \mathcal{Y}, \exists$ a constant $0<C_{1}<\infty$ with

$$
\left|\left(\mathcal{O} y_{0}^{\prime}\right)(t)-y_{0}^{\prime}(t)\right|\left|\mathcal{V}(t, y(t))\left[\frac{1}{\Gamma(q)} \int_{0}^{t}(t-\xi)^{q-1} Q_{\alpha, \beta, \delta}^{\gamma, q, r}(t-\xi)^{q} \mathcal{W}(\xi, y(\xi)) \mathrm{d} \xi\right]-y_{0}^{\prime}(t)\right| \leq C_{1} \varepsilon,
$$

$\forall t \in[0, a]$. so $\hat{\rho}\left(\mathcal{O}\left(y_{0}^{\prime}\right), y_{0}^{\prime}\right)<\infty$. Therefore, Theorem 2.1(I) indicates that $\exists$ a CF: $y_{0}^{\prime}:[0, a] \rightarrow \mathbf{R}$ such that $\mathcal{O}^{n} y_{0}^{\prime} \rightarrow y_{0}^{\prime}$ in ( $y, \hat{\rho})$ as $n \rightarrow \infty, y_{0}^{\prime}=\mathcal{O}\left(y_{0}^{\prime}\right)$ where $y_{0}^{\prime}$ satisfies equation (1.1) for any $t \in J$. If $y \in \mathcal{y}$, then $y_{0}^{\prime}$ and $y$ are CFs defined on a compact interval $[0, a]$. Thus, $\exists$ a constant $C_{y}>0$ with

$$
\left|y_{0}^{\prime}(t)-y(t)\right| \leq C_{x} \varepsilon
$$

$\forall t \in[0, a]$. This indicates that $\hat{\rho}\left(y_{0}^{\prime}, y\right)<\infty$ for every $y \in y$ or equivalently

$$
\left\{y \in y: \hat{\rho}\left(y_{0}^{\prime}, y\right)<\infty\right\}=y \text {. }
$$


Hence, from Theorem 2.1(II) $y_{0}^{\prime}$ is a unique continuous function (UqCF) with property (1.1). Also, it implies from (3.1)

$$
\hat{\rho}(\mathcal{O}(y(t)), y(t)) \leq \varepsilon,
$$

$\forall t \in[0, a]$. At last,

$$
\hat{\rho}\left(y, y_{0}^{\prime}\right) \leq \frac{1}{1-K^{\prime}} \hat{\rho}(\mathcal{O} y, y) \leq \frac{1}{1-K^{\prime}} \varepsilon .
$$

This means that the quadratic FIE is Hyers-Ulam stable.

The HURS of equation (1.1) is investigated below.

Theorem 3.3. For a closed and bounded interval $J=[0, a]$ of the real line $\mathbb{R}$ for some $a>0$, suppose that $\mathcal{V}, \mathcal{W}: J \times \mathbb{R} \rightarrow \mathbb{R}$ are CFs, $q \in[1,2)$ and a gamma function, denoted by $\Gamma$, the following are satisfied:

$$
|\mathcal{V}(t, y(t))-\mathcal{V}(t, u(t))| \leq M_{v}|y(t)-u(t)|
$$

and

$$
|\mathcal{W}(t, y(t))-\mathcal{W}(t, u(t))| \leq M_{w}|y(t)-u(t)|
$$

and

$$
\left[\int_{0}^{t}(\varphi(\xi))^{1 / p} \mathrm{~d} \xi\right]^{p} \leq C \varphi(t)
$$

for any $t \in J, y, u \in \mathbb{R}$ and suppose that

$$
\left|y(t)-\mathcal{V}(t, y(t))\left[\frac{1}{\Gamma(q)} \int_{0}^{t}(t-\xi)^{q-1} Q_{\alpha, \beta, \delta}^{\gamma, q, r}(t-\xi)^{q} \mathcal{W}(\xi, y(\xi)) \mathrm{d} \xi\right]\right| \leq \varepsilon \varphi(t),
$$

and we also suppose that

$$
\begin{aligned}
0< & \left(M_{v} M_{w} K C \varepsilon \varphi(t)+M_{w} C\|\mathcal{V}\|\right) \frac{1}{\Gamma(q)} \sum_{m=0}^{\infty}\left[\frac{\prod_{n=1}^{r} \beta\left(b_{n}, m\right)(\gamma)_{q m}}{\prod_{n=1}^{r} \beta\left(a_{n}, m\right)(\delta)_{q m} \Gamma(\beta+\alpha m)}\right]^{1 / w}\left(\frac{w t^{\frac{m q+q+w-1}{w}}}{m q+q+w-1}\right) \\
& +M_{v}\|\mathcal{W}\| \frac{1}{\Gamma(q)} \sum_{m=0}^{\infty} \frac{\prod_{n=1}^{r} \beta\left(b_{n}, m\right)(\gamma)_{q m}}{\prod_{n=1}^{r} \beta\left(a_{n}, m\right)(\delta)_{q m} \Gamma(\beta+\alpha m)} \cdot\left(\frac{t^{q(m+1)}}{q(m+1)}\right) \\
= & K^{\prime}<1 .
\end{aligned}
$$

Then, the quadratic FIE is Hyers-Ulam stable.

Proof. Let us consider the continuous functions space: $y=C([0, a], \mathbb{R})$ and $g \in y$, with a GMr, expressed as:

$$
\hat{\rho}(g, h)=\inf \{K \in[0, \infty]:|g(t)-h(t)| \leq K \varepsilon \varphi(t), \forall t \in J\} .
$$

Obviously, $(y, \hat{\rho})$ is a GCMSp. Let us now form an operator: $\mathcal{O}: y \rightarrow y$ as:

$$
\mathcal{O}(y(t))=\mathcal{V}(t, y(t))\left[\frac{1}{\Gamma(q)} \int_{0}^{t}(t-\xi)^{q-1} Q_{\alpha, \beta, \delta}^{\gamma, q, r}(t-\xi)^{q} \mathcal{W}(\xi, y(\xi)) \mathrm{d} \xi\right] .
$$

From the definition $\mathcal{O}$ and equations (3.5) and (3.6), we get:

$$
\begin{aligned}
|\mathcal{O}(y(t))-\mathcal{O}(u(t))|= & \mid \mathcal{V}(t, y(t))\left[\frac{1}{\Gamma(q)} \int_{0}^{t}(t-\xi)^{q-1} Q_{\alpha, \beta, \delta}^{\gamma, q, r}(t-\xi)^{q} \mathcal{W}(\xi, y(\xi)) \mathrm{d} \xi\right] \\
& -\mathcal{V}(t, u(t))\left[\frac{1}{\Gamma(q)} \int_{0}^{t}(t-\xi)^{q-1} Q_{\alpha, \beta, \delta}^{\gamma, q, r}(t-\xi)^{q} \mathcal{W}(\xi, u(\xi)) \mathrm{d} \xi\right] \mid
\end{aligned}
$$




$$
\begin{aligned}
& \leq|\mathcal{V}(t, y(t))-\mathcal{V}(t, u(t))|\left[\frac{1}{\Gamma(q)} \int_{0}^{t}(t-\xi)^{q-1} Q_{\alpha, \beta, \delta}^{\gamma, q, r}(t-\xi)^{q}|\mathcal{W}(\xi, y(\xi))-\mathcal{W}(\xi, u(\xi))| \mathrm{d} \xi\right] \\
& +|\mathcal{V}(t, y(t))-\mathcal{V}(t, u(t))|\left[\frac{1}{\Gamma(q)} \int_{0}^{t}(t-\xi)^{q-1} Q_{\alpha, \beta, \delta}^{\gamma, q, r}(t-\xi)^{q}|\mathcal{W}(\xi, u(\xi))| \mathrm{d} \xi\right] \\
& +|\mathcal{V}(t, u(t))|\left[\frac{1}{\Gamma(q)} \int_{0}^{t}(t-\xi)^{q-1} Q_{\alpha, \beta, \delta}^{\gamma, q, r}(t-\xi)^{q}|\mathcal{W}(\xi, y(\xi))-\mathcal{W}(\xi, u(\xi))| \mathrm{d} \xi\right] \\
& \leq M_{v}|y(t)-u(t)|\left[\frac{1}{\Gamma(q)} \int_{0}^{t}(t-\xi)^{q-1} Q_{\alpha, \beta, \delta}^{\gamma, q, r}(t-\xi)^{q} M_{w}|y(\xi)-u(\xi)| \mathrm{d} \xi\right] \\
& +M_{v}|y(t)-u(t)|\left[\frac{1}{\Gamma(q)} \int_{0}^{t}(t-\xi)^{q-1} Q_{\alpha, \beta, \delta}^{\gamma, q, r}(t-\xi)^{q}\|\mathcal{W}\| \mathrm{d} \xi\right] \\
& +\|\mathcal{V}\|\left[\frac{1}{\Gamma(q)} \int_{0}^{t}(t-\xi)^{q-1} Q_{\alpha, \beta, \delta}^{\gamma, q, r}(t-\xi)^{q} M_{V}|y(\xi)-u(\xi)| \mathrm{d} \xi\right] \\
& \leq M_{v} M_{w} K^{2} \varepsilon^{2} \varphi(t)\left[\frac{1}{\Gamma(q)} \int_{0}^{t}(t-\xi)^{q-1} Q_{\alpha, \beta, \delta}^{\gamma, q, r}(t-\xi)^{q} \varphi(\xi) \mathrm{d} \xi\right] \\
& +M_{v} K \varepsilon\|\mathcal{W}\| \varphi(t)\left[\frac{1}{\Gamma(q)} \int_{0}^{t}(t-\xi)^{q-1} Q_{\alpha, \beta, \delta}^{\gamma, q, r}(t-\xi)^{q} \mathrm{~d} \xi\right] \\
& +M_{\nu} K \varepsilon\|\mathcal{V}\|\left[\frac{1}{\Gamma(q)} \int_{0}^{t}(t-\xi)^{q-1} Q_{\alpha, \beta, \delta}^{\gamma, q, r}(t-\xi)^{q} \varphi(\xi) \mathrm{d} \xi\right] \\
& \leq\left(M_{v} M_{w} K^{2} \varepsilon^{2} \varphi(t)+M_{v} K \varepsilon\|\mathcal{V}\|\right)\left[\frac{1}{\Gamma(q)} \int_{0}^{t}(t-\xi)^{q-1} Q_{\alpha, \beta, \delta}^{\gamma, q, r}(t-\xi)^{q} \varphi(\xi) \mathrm{d} \xi\right] \\
& +M_{v} K \varepsilon\|\mathcal{W}\| \varphi(t)\left[\frac{1}{\Gamma(q)} \int_{0}^{t}(t-\xi)^{q-1} Q_{\alpha, \beta, \delta}^{\gamma, q, r}(t-\xi)^{q} \mathrm{~d} \xi\right] \\
& \leq\left(M_{v} M_{w} K^{2} \varepsilon^{2} \varphi(t)+M_{v} K \varepsilon\|\mathcal{V}\|\right) \frac{1}{\Gamma(q)}\left[\int_{0}^{t}(t-\xi)^{q-1} Q_{\alpha, \beta, \delta}^{\gamma, q, r}\left((t-\xi)^{q}\right)^{1 / w} \mathrm{~d} \xi\right]^{w} \\
& \times\left[\int_{0}^{t}(\varphi(\xi))^{1 / p} \mathrm{~d} \xi\right]^{p} \\
& +M_{v} K \varepsilon\|\mathcal{W}\|\left[\frac{1}{\Gamma(q)} \int_{0}^{t}(t-\xi)^{q-1} Q_{\alpha, \beta, \delta}^{\gamma, q, r}(t-\xi)^{q} \mathrm{~d} \xi\right] \\
& \leq\left(M_{v} M_{w} K^{2} \varepsilon^{2} \varphi(t)+M_{v} K \varepsilon\|\mathcal{V}\|\right) C \varphi(t) \frac{1}{\Gamma(q)} \int_{0}^{t}(t-\xi)^{(q-1) / w} \\
& \times \sum_{m=0}^{\infty}\left[\frac{\prod_{n=1}^{r} \beta\left(b_{n}, m\right)(\gamma)_{q m}}{\prod_{n=1}^{r} \beta\left(a_{n}, m\right)(\delta)_{q m} \Gamma(\beta+\alpha m)}\right]^{1 / w}\left((t-\xi)^{q}\right)^{m q / w} \mathrm{~d} \xi \\
& +M_{v} K \varepsilon\|\mathcal{W}\| \varphi(t) \frac{1}{\Gamma(q)} \int_{0}^{t}(t-\xi)^{q-1}
\end{aligned}
$$




$$
\begin{aligned}
& \times \sum_{m=0}^{\infty}\left[\frac{\prod_{n=1}^{r} \beta\left(b_{n}, m\right)(\gamma)_{q m}}{\prod_{n=1}^{r} \beta\left(a_{n}, m\right)(\delta)_{q m} \Gamma(\beta+\alpha m)}\right]^{1 / w}(t-\xi)^{m q} \mathrm{~d} \xi \\
\leq & \left(M_{v} M_{w} K^{2} \varepsilon^{2} \varphi(t)+M_{v} K \varepsilon\|\mathcal{V}\|\right) C \varphi(t) \frac{1}{\Gamma(q)} \sum_{m=0}^{\infty}\left[\frac{\prod_{n=1}^{r} \beta\left(b_{n}, m\right)(\gamma)_{q m}}{\prod_{n=1}^{r} \beta\left(a_{n}, m\right)(\delta)_{q m} \Gamma(\beta+\alpha m)}\right]^{1 / w} \\
& \times \int_{0}^{t}(t-\xi)^{(m q+q-1) / w} \mathrm{~d} \xi \\
& +M_{v} K \varepsilon\|\mathcal{W}\| \varphi(t) \frac{1}{\Gamma(q)} \sum_{m=0}^{\infty} \frac{\prod_{n=1}^{r} \beta\left(b_{n}, m\right)(\gamma)_{q m}}{\prod_{n=1}^{r} \beta\left(a_{n}, m\right)(\delta)_{q m} \Gamma(\beta+\alpha m)} \int_{0}^{t}(t-\xi)^{m q+q-1} \mathrm{~d} \xi \\
\leq & \left(M_{v} M_{w} K^{2} \varepsilon^{2} \varphi(t)+M_{v} K \varepsilon\|\mathcal{V}\|\right) C \varphi(t) \frac{1}{\Gamma(q)} \sum_{m=0}^{\infty}\left[\frac{\prod_{n=1}^{r} \beta\left(b_{n}, m\right)(\gamma)_{q m}}{\prod_{n=1}^{r} \beta\left(a_{n}, m\right)(\delta)_{q m} \Gamma(\beta+\alpha m)}\right]^{1 / w} \\
& \times \frac{w}{m q+q+q-1} t^{(m q+q+w-1) / w} \\
& +M_{v} K \varepsilon\|\mathcal{W}\| \varphi(t) \frac{1}{\Gamma(q)} \sum_{m=0}^{\infty} \frac{\prod_{n=1}^{r} \beta\left(b_{n}, m\right)(\gamma)_{q m}}{\prod_{n=1}^{r} \beta\left(a_{n}, m\right)(\delta)_{q m} \Gamma(\beta+\alpha m)} \frac{t^{(m+1) q}}{(m+1) q} \\
\leq & K \varepsilon \varphi(t) K^{\prime} .
\end{aligned}
$$

Note that $0<K^{\prime}<1$. We conclude that $\mathcal{O}$ is contraction mapping (CoMp). Let us take $y_{0}^{\prime} \in \mathcal{y}$, from the continuous property of $y_{0}^{\prime} \in \mathcal{y}$ and $\mathcal{O}\left(y_{0}^{\prime}\right) \in y \exists$ a constant $0<C_{1}<\infty$ with

$$
\begin{aligned}
\left|\mathcal{O}\left(y_{0}^{\prime}\right)(t)-y_{0}^{\prime}(t)\right| & =\left|\mathcal{V}(t, y(t))\left[\frac{1}{\Gamma(q)} \int_{0}^{t}(t-\xi)^{q-1} Q_{\alpha, \beta, \delta}^{\gamma, q, r}(t-\xi)^{q} \mathcal{W}(\xi, y(\xi)) \mathrm{d} \xi\right]-y_{0}^{\prime}(t)\right| \\
& \leq C_{1} \varepsilon \varphi(t),
\end{aligned}
$$

$\forall t \in[0, a]$. So, $\hat{\rho}\left(\mathcal{O}\left(y_{0}\right), y_{0}^{\prime}\right)<\infty$. Thus, Theorem 2.1(a) indicates that $\exists$ a CF: $u_{0}^{\prime}:[0, a] \rightarrow \mathbb{R}$ such that $\mathcal{O}^{n}\left(y_{0}\right) \rightarrow u_{0}^{\prime}$ in $(y, \hat{\rho})$ as $n \rightarrow \infty, u_{0}^{\prime}=\mathcal{O}\left(u_{0}^{\prime}\right)$; therefore, $u_{0}^{\prime}$ satisfies equation (1.1) for any $t \in J$. If $y \in y$, then $y_{0}^{\prime}$ and $y$ are CFs defined on a compact interval $[0, a]$. Thus, $\exists$ a constant $C_{x}>0$ with

$$
\left|y_{0}^{\prime}(t)-y(t)\right| \leq C_{y} \varepsilon \varphi(t), \quad \forall t \in[0, a] .
$$

This indicates that $\hat{\rho}\left(y_{0}^{\prime}, y\right)<\infty$ for every $y \in y$ or equivalently $\left\{y \in y: \hat{\rho}\left(y_{0}^{\prime}, y\right)<\infty\right\}=y$. Hence, from Theorem (2.1)(II) $u_{0}^{\prime}$ is a UqCF with property (1.1). As a result, from (3.7), it implies that

$$
\hat{\rho}(\mathcal{O}(u(t)), u(t)) \leq \varepsilon \varphi(t),
$$

$\forall t \in[0, a]$. At last,

$$
\hat{\rho}\left(u, u_{0}^{\prime}\right) \leq \frac{1}{1-K^{\prime}} \hat{\rho}(\mathcal{O}(u), u) \leq \frac{1}{1-K^{\prime}} \varepsilon \varphi(t) .
$$

Thus, the quadratic FIE is Hyers-Ulam-Rassias stable.

\section{ML-Hyers-Ulam Stability}

The ML-HUS of equation (1.1) is studied in this section.

Definition 4.1. If for each function y satisfies

$$
\left|y(t)-\mathcal{V}(t, y(t))\left[\frac{1}{\Gamma(q)} \int_{0}^{t}(t-\xi)^{q-1} Q_{\alpha, \beta, \delta}^{\gamma, q, r}(t-\xi)^{q} \mathcal{W}(\xi, y(\xi)) \mathrm{d} \xi\right]\right| \leq \varepsilon E_{q}\left(t^{q}\right),
$$


$\exists$ an equation's (1.1) solution $y_{0}$, and a constant, $c>0$, which is an independent of both $y$ and $y_{0}$ such that

$$
\left|y(t)-y_{0}(t)\right| \leq c \varepsilon E_{q}\left(t^{q}\right),
$$

for each $t \in[0, a]$, then equation (1.1) is named as $M L-\mathcal{H}$ yers-Ulam stable.

Theorem 4.2. For a closed and bounded interval $J=[0, a]$ of the real line $\mathbb{R}$ for some $a>0$, suppose that $\mathcal{V}, \mathcal{W}: J \times \mathbf{R} \rightarrow \mathbb{R}$ are continuous functions, $q \in[1,2)$ and a gamma function, denoted by $\Gamma$, the following are satisfied:

$$
|\mathcal{V}(t, y(t))-\mathcal{W}(t, u(t))| \leq M_{v}|y(t)-u(t)|
$$

and

$$
|\mathcal{W}(t, y(t))-\mathcal{W}(t, u(t))| \leq M_{w}|y(t)-u(t)|,
$$

for any $t \in J, y, u \in \mathbb{R}$, and suppose that

$$
\left.\mid y(t)-\mathcal{V}(t, y(t))\left[\frac{1}{\Gamma(q)} \int_{0}^{t}(t-\xi)^{q-1} Q_{\alpha, \beta, \delta}^{\gamma, q, r}(t-\xi)^{q} \mathcal{W}(\xi, y(\xi)) \mathrm{d} \xi\right)\right] \mid \leq \varepsilon E_{q}\left(t^{q}\right) .
$$

Also, suppose that

$$
\begin{aligned}
0< & \left(M_{v} M_{w} K \varepsilon E_{q}\left(t^{q}\right)+M_{w} K \varepsilon\|\mathcal{V}\|\right) \\
& \times \frac{1}{\Gamma(q)} \sum_{m=0}^{\infty} \frac{\prod_{n=1}^{r} \beta\left(b_{n}, m\right)(\gamma)_{q m}}{\prod_{n=1}^{r} \beta\left(a_{n}, m\right)(\delta)_{q m} \Gamma(\beta+\alpha m)} \Gamma((m+1) q) \\
& +M_{v}\|\mathcal{W}\| \frac{1}{\Gamma(q)} \sum_{m=0}^{\infty} \frac{\prod_{n=1}^{r} \beta\left(b_{n}, m\right)(\gamma)_{q m}}{\prod_{n=1}^{r} \beta\left(a_{n}, m\right)(\delta)_{q m} \Gamma(\beta+\alpha m)}\left(\frac{t^{q(m+1)}}{q(m+1)}\right) \\
= & K^{\prime}<1,
\end{aligned}
$$

then quadratic FIE is Hyers-Ulam stable.

Proof. Let us consider the continuous functions space: $y=C([0, a], \mathbb{R})$ and $g \in y$, with a GMr, expressed as:

$$
\hat{\rho}(g, h)=\inf \left\{K \in[0, \infty]:|g(x)-h(x)| \leq K \varepsilon E_{q}\left(t^{q}\right), \forall t \in J\right\} .
$$

Obviously, $(y, \hat{\rho})$ is a GCMSp. Let us express an operator: $\mathcal{O}: y \rightarrow y$ by

$$
\mathcal{O}(y(t))=\mathcal{V}(t, y(t))\left[\frac{1}{\Gamma(q)} \int_{0}^{t}(t-\xi)^{q-1} Q_{\alpha, \beta, \delta}^{\gamma, q, r}(t-\xi)^{q} \mathcal{W}(\xi, y(\xi)) \mathrm{d} \xi\right] .
$$

From the definition $\mathcal{O}$ and equations (4.1) and (4.2), we get:

$$
\begin{aligned}
|\mathcal{O}(y(t))-\mathcal{O}(u(t))|= & \mid \mathcal{V}(t, y(t))\left[\frac{1}{\Gamma(q)} \int_{0}^{t}(t-\xi)^{q-1} Q_{\alpha, \beta, \delta}^{\gamma, q, r}(t-\xi)^{q} \mathcal{W}(\xi, y(\xi)) \mathrm{d} \xi\right] \\
& -\mathcal{V}(t, u(t))\left[\frac{1}{\Gamma(q)} \int_{0}^{t}(t-\xi)^{q-1} Q_{\alpha, \beta, \delta}^{\gamma, q, r}(t-\xi)^{q} \mathcal{W}(\xi, u(\xi)) \mathrm{d} \xi\right] \mid \\
\leq & |\mathcal{V}(t, y(t))-\mathcal{V}(t, u(t))| \\
& \times\left[\frac{1}{\Gamma(q)} \int_{0}^{t}(t-\xi)^{q-1} Q_{\alpha, \beta, \delta}^{\gamma, q, r}(t-\xi)^{q}|\mathcal{W}(\xi, y(\xi))-\mathcal{W}(\xi, u(\xi))| \mathrm{d} \xi\right] \\
& +|\mathcal{V}(t, y(t))-\mathcal{V}(t, u(t))|
\end{aligned}
$$




$$
\begin{aligned}
& \times\left[\frac{1}{\Gamma(q)} \int_{0}^{t}(t-\xi)^{q-1} Q_{\alpha, \beta, \delta}^{\gamma, q, r}(t-\xi)^{q}|\mathcal{W}(\xi, u(\xi))| \mathrm{d} \xi\right] \\
& +|\mathcal{V}(t, u(t))| \\
& \times\left[\frac{1}{\Gamma(q)} \int_{0}^{t}(t-\xi)^{q-1} Q_{\alpha, \beta, \delta}^{\gamma, q, r}(t-\xi)^{q}|\mathcal{W}(\xi, y(\xi))-\mathcal{W}(\xi, u(\xi))| \mathrm{d} \xi\right] \\
& \leq M_{v}|y(t)-u(t)| \\
& \times\left[\frac{1}{\Gamma(q)} \int_{0}^{t}(t-\xi)^{q-1} Q_{\alpha, \beta, \delta}^{\gamma, q, r}(t-\xi)^{q} M_{w}|y(\xi)-u(\xi)| \mathrm{d} \xi\right] \\
& +M_{v}|y(t)-u(t)| \\
& \times\left[\frac{1}{\Gamma(q)} \int_{0}^{t}(t-\xi)^{q-1} Q_{\alpha, \beta, \delta}^{\gamma, q, r}(t-\xi)^{q}\|\mathcal{W}\| \mathrm{d} \xi\right] \\
& +\|\mathcal{V}\|\left[\frac{1}{\Gamma(q)} \int_{0}^{t}(t-\xi)^{q-1} Q_{\alpha, \beta, \delta}^{\gamma, q, r}(t-\xi)^{q} M_{V}|y(\xi)-u(\xi)| \mathrm{d} \xi\right] \\
& \leq M_{v} M_{w} K^{2} \varepsilon^{2} E_{q}\left(t^{q}\right) \\
& \times\left[\frac{1}{\Gamma(q)} \int_{0}^{t}(t-\xi)^{q-1} Q_{\alpha, \beta, \delta}^{\gamma, q, r}(t-\xi)^{q} E_{q}\left(\xi^{q}\right) \mathrm{d} \xi\right] \\
& +M_{v} K \varepsilon\|\mathcal{W}\| E_{q}\left(t^{q}\right)\left[\frac{1}{\Gamma(q)} \int_{0}^{t}(t-\xi)^{q-1} Q_{\alpha, \beta, \delta}^{\gamma, q, r}(t-\xi)^{q} \mathrm{~d} \xi\right] \\
& +M_{v} K \varepsilon\|\mathcal{V}\|\left[\frac{1}{\Gamma(q)} \int_{0}^{t}(t-\xi)^{q-1} Q_{\alpha, \beta, \delta}^{\gamma, q, r}(t-\xi)^{q} E_{q}\left(\xi^{q}\right) \mathrm{d} \xi\right] \\
& \leq\left(M_{v} M_{w} K^{2} \varepsilon^{2} E_{q}\left(t^{q}\right)+M_{v} K \varepsilon\|\mathcal{V}\|\right) \\
& \times\left[\frac{1}{\Gamma(q)} \int_{0}^{t}(t-\xi)^{q-1} Q_{\alpha, \beta, \delta}^{\gamma, q, r}(t-\xi)^{q} E_{q}\left(\xi^{q}\right) \mathrm{d} \xi\right] \\
& +M_{v} K \varepsilon\|\mathcal{W}\| E_{q}\left(t^{q}\right)\left[\frac{1}{\Gamma(q)} \int_{0}^{t}(t-\xi)^{q-1} Q_{\alpha, \beta, \delta}^{\gamma, q, r}(t-\xi)^{q} \mathrm{~d} \xi\right] \\
& \leq\left(M_{v} M_{w} K^{2} \varepsilon^{2} E_{q}\left(t^{q}\right)+M_{v} K \varepsilon\|\mathcal{V}\|\right) \\
& \times \frac{1}{\Gamma(q)}\left[\int_{0}^{t}(t-\xi)^{q-1} \sum_{m=0}^{\infty} \frac{\prod_{n=1}^{r} \beta\left(b_{n}, m\right)(\gamma)_{q m}}{\prod_{n=1}^{r} \beta\left(a_{n}, m\right)(\delta)_{q m} \Gamma(\beta+\alpha m)}\right. \\
& \times(t-\xi)^{m q} \sum_{n=0}^{\infty} \frac{s^{n q}}{\Gamma(q n+1)} \mathrm{d} \xi \\
& +M_{v} K \varepsilon\|\mathcal{W}\| E_{q}\left(t^{q}\right)\left[\frac{1}{\Gamma(q)} \int_{0}^{t}(t-\xi)^{q-1}\right. \\
& \times \sum_{m=0}^{\infty} \frac{\prod_{n=1}^{r} \beta\left(b_{n}, m\right)(\gamma)_{q m}}{\prod_{n=1}^{r} \beta\left(a_{n}, m\right)(\delta)_{q m} \Gamma(\beta+\alpha m)} \mathrm{d} \xi
\end{aligned}
$$




$$
\begin{aligned}
& \leq\left(M_{v} M_{w} K^{2} \varepsilon^{2} E_{q}\left(t^{q}\right)+M_{v} K \varepsilon\|\mathcal{V}\|\right) \\
& \times \frac{1}{\Gamma(q)} \sum_{m=0}^{\infty} \frac{\prod_{n=1}^{r} \beta\left(b_{n}, m\right)(\gamma)_{q m}}{\prod_{n=1}^{r} \beta\left(a_{n}, m\right)(\delta)_{q m} \Gamma(\beta+\alpha m)} \\
& \times \sum_{n=0}^{\infty} \frac{s^{n q}}{\Gamma(q n+1)} \int_{0}^{t}(t-\xi)^{q-1}(t-\xi)^{m q} \xi^{n q} \mathrm{~d} \xi \\
& +M_{v} K \varepsilon\|\mathcal{W}\| E_{q}\left(t^{q}\right) \frac{1}{\Gamma(q)} \int_{0}^{t}(t-\xi)^{q-1} \\
& \times \sum_{m=0}^{\infty} \frac{\prod_{n=1}^{r} \beta\left(b_{n}, m\right)(\gamma)_{q m}}{\prod_{n=1}^{r} \beta\left(a_{n}, m\right)(\delta)_{q m} \Gamma(\beta+\alpha m)}(t-\xi)^{m q} \mathrm{~d} \xi \\
& \leq\left(M_{v} M_{w} K^{2} \varepsilon^{2} E_{q}\left(t^{q}\right)+M_{v} K \varepsilon\|\mathcal{V}\|\right) \\
& \times \frac{1}{\Gamma(q)} \sum_{m=0}^{\infty} \frac{\prod_{n=1}^{r} \beta\left(b_{n}, m\right)(\gamma)_{q m}}{\prod_{n=1}^{r} \beta\left(a_{n}, m\right)(\delta)_{q m} \Gamma(\beta+\alpha m)} \\
& \times \sum_{n=0}^{\infty} \frac{s^{n q}}{\Gamma(q n+1)} \int_{0}^{t}(t-\xi)^{m q+q-1} s^{n q} \mathrm{~d} \xi \\
& +M_{v} K \varepsilon\|\mathcal{W}\| E_{q}\left(t^{q}\right) \frac{1}{\Gamma(q)} \\
& \times \sum_{m=0}^{\infty} \frac{\prod_{n=1}^{r} \beta\left(b_{n}, m\right)(\gamma)_{q m}}{\prod_{n=1}^{r} \beta\left(a_{n}, m\right)(\delta)_{q m} \Gamma(\beta+\alpha m)} \\
& \times \int_{0}^{t}(t-\xi)^{m q+q-1} \mathrm{~d} \xi \\
& \leq\left(M_{v} M_{w} K^{2} \varepsilon^{2} E_{q}\left(t^{q}\right)+M_{w} K \varepsilon\|\mathcal{V}\|\right) \\
& \times \frac{1}{\Gamma(q)} \sum_{m=0}^{\infty} \frac{\prod_{n=1}^{r} \beta\left(b_{n}, m\right)(\gamma)_{q m}}{\prod_{n=1}^{r} \beta\left(a_{n}, m\right)(\delta)_{q m} \Gamma(\beta+\alpha m)} \\
& \left.\times \Gamma((m+1) q) \sum_{s=0}^{\infty} \frac{1}{\Gamma(q s+1)} t^{s q}\right) \\
& +M_{v} K \varepsilon\|\mathcal{W}\| E_{q}\left(t^{q}\right) \\
& \times \frac{1}{\Gamma(q)} \sum_{m=0}^{\infty} \frac{\prod_{n=1}^{r} \beta\left(b_{n}, m\right)(\gamma)_{q m}}{\prod_{n=1}^{r} \beta\left(a_{n}, m\right)(\delta)_{q m} \Gamma(\beta+\alpha m)}\left(\frac{t^{(m+1) q}}{(m+1) q}\right) \\
& \leq\left(M_{v} M_{w} K^{2} \varepsilon^{2} E_{q}\left(t^{q}\right)+M_{w} K \varepsilon\|\mathcal{V}\|\right) \\
& \times \frac{1}{\Gamma(q)} \sum_{m=0}^{\infty} \frac{\prod_{n=1}^{r} \beta\left(b_{n}, m\right)(\gamma)_{q m}}{\prod_{n=1}^{r} \beta\left(a_{n}, m\right)(\delta)_{q m} \Gamma(\beta+\alpha m)} \Gamma((m+1) q) E_{q}\left(t^{q}\right) \\
& +M_{v} K \varepsilon\|\mathcal{W}\| E_{q}\left(t^{q}\right) \\
& \times \frac{1}{\Gamma(q)} \sum_{m=0}^{\infty} \frac{\prod_{n=1}^{r} \beta\left(b_{n}, m\right)(\gamma)_{q m}}{\prod_{n=1}^{r} \beta\left(a_{n}, m\right)(\delta)_{q m} \Gamma(\beta+\alpha m)}\left(\frac{t^{(m+1) q}}{(m+1) q}\right) \\
& \leq K \varepsilon E_{q}\left(t^{q}\right) K^{\prime} .
\end{aligned}
$$


We note that $0<K^{\prime}<1$. We conclude that $\mathcal{O}$ is contraction mapping. Let us take $y_{0}^{\prime} \in \mathcal{y}$, from the continuous property of $y_{0}^{\prime} \in \mathcal{Y}$ and $\mathcal{O}\left(y_{0}^{\prime}\right) \in \mathcal{y}, \exists$ a constant $0<C_{1}<\infty$ with

$$
\begin{aligned}
\left|\mathcal{O}\left(y_{0}^{\prime}\right)(t)-y_{0}^{\prime}(t)\right| & =\left|\mathcal{V}(t, y(t))\left[\frac{1}{\Gamma(q)} \int_{0}^{t}(t-\xi)^{q-1} Q_{\alpha, \beta, \delta}^{\gamma, q, r}(t-\xi)^{q} \mathcal{W}(\xi, y(\xi)) \mathrm{d} \xi\right]-y_{0}^{\prime}(t)\right| \\
& \leq C_{1} \varepsilon E_{q}\left(t^{q}\right),
\end{aligned}
$$

$\forall t \in[0, a]$. So, $\hat{\rho}\left(\mathcal{O}\left(y_{0}^{\prime}\right), y_{0}^{\prime}\right)<\infty$. Thus, Theorem 2.1(I) indicates that $\exists$ a CF: $u_{0}^{\prime}:[0, a] \rightarrow \mathbb{R} \ni \mathcal{O}^{n} y_{0}^{\prime} \rightarrow u_{0}^{\prime}$ in $(y, \hat{\rho})$ as $n \rightarrow \infty, u_{0}^{\prime}=\mathcal{O}\left(u_{0}^{\prime}\right)$; therefore, $u_{0}^{\prime}$ satisfies equation (1.1) for any $t \in J$. If $y \in y$, then $y_{0}^{\prime}$ and $y$ are CFs defined on a compact interval $[0, a]$. Thus, $\exists$ a constant $C_{y}>0$ with

$$
\left|y_{0}^{\prime}(t)-y(t)\right| \leq C_{y} \varepsilon \mathcal{E}_{q}\left(t^{q}\right),
$$

$\forall t \in[0, a]$. This indicates that $\hat{\rho}\left(y_{0}^{\prime}, y\right)<\infty$ for every $y \in y$ or equivalently

$$
\left\{y \in y: \hat{\rho}\left(y_{0}^{\prime}, y\right)<\infty\right\}=y .
$$

Hence, from Theorem (2.1)(II) $u_{0}^{\prime}$ is a UqCF with property (1.1). From (4.3), it implies that

$$
\hat{\rho}(\mathcal{O}(u(t)), u(t)) \leq \varepsilon \mathcal{E}_{q}\left(t^{q}\right),
$$

$\forall t \in[0, a]$. At last,

$$
\hat{\rho}\left(u, u_{0}^{\prime}\right) \leq \frac{1}{1-K^{\prime}} \hat{\rho}(\mathcal{O}(u), u) \leq \frac{1}{1-K^{\prime}} \varepsilon \mathcal{E}_{q}\left(t^{q}\right) .
$$

This means that the quadratic FIE is ML-Hyers-Ulam stable.

\section{Conclusion}

Functional equations, particularly quadratic fractional integral equations, have been applied in inner product spaces' characterization. An essential parallelogram equality:

$$
\|y+z\|^{2}+\|y+z\|^{2}=2\left(\|y\|^{2}+\|z\|^{2}\right),
$$

is satisfied by a square norm on an inner product space. Both of the HUS and ML-HURS have been investigated for the proposed DEq. All obtained results are new which show that ML is very important in proving differential equation's stability. Various differential equations' classes can been unified via our new generalized technique which can inspire interested engineers and scientists to work on future research studies, particularly in the field of environmental sciences, with other related applications based on this technique, and this technique will be extended further in our future research works by working on modeling various scientific phenomena.

Funding information: The authors state no funding involved.

Author contributions: All authors have accepted responsibility for the entire content of this manuscript and approved its submission.

Conflict of interest: The authors state no conflict of interest.

\section{References}

[1] Argyros IK. Quadratic equations and applications to Chandrasekhar's and related equations. Bull Aust Math Soc. 1985;32:275-292. 
[2] Busbridge LW. The Mathematics of Radiative Transfer. Cambridge University Press: Cambridge, MA; 1960.

[3] Hyers DH. On the stability of the linear functional equation. Proc Nat Acad Sci. 1941;27:222-224.

[4] Rassias TM. On the stability of linear mapping in Banach spaces. Proc Amer Math Soc. 1978;72:297-300.

[5] Rassias JM. On approximation of approximately linear mappings by linear mappings. J Func Anal. 1982;46(1):126-130.

[6] C. Alsina and R. Ger. On some inequalities and stability results related to the exponential function. J Inequal Appl. 1998;2:373-380.

[7] Ibrahim RW. Ulam stability for fractional differential equation in complex domain. Abstr Appl Anal. 2012;2012:1-8.

[8] Wang JR, Zhou Y, Medvěd M. Existence and stability of fractional differential equations with Hadamard derivative. Topol Meth Nonl Anal. 2013;41:113-133.

[9] Eghbali N, Kalvandi V, Rassias JM. A fixed point approach to the Mittag-Leffler-Hyers-Ulam stability of a fractional integral equation. Open Math. 2016;14:237-246.

[10] Beyer H, Kempfle S. Definition of physical consistent damping laws with fractional derivatives. Z Angew Math Mech. 1995;75:623-635.

[11] He JH. Approximate analytic solution for seepage flow with fractional derivatives in porous media. Comput Method Appl M. 1998;167:57-68.

[12] Abbas MI, Ragusa MA. Solvability of Langevin equations with two Hadamard fractional derivatives via Mittag-Leffler functions. Appl Anal. 2020:1-15.

[13] Jung CY, Farid G, Mahreen K, Shim SH. Inequalities for a Unified Integral Operator for Strongly $\alpha$, m-Convex Function and Related Results in Fractional Calculus. J Funct Spaces. 2021;2021:1-8.

[14] Farid G, Mubeen S, Set E. Fractional inequalities associated with a generalized Mittag-Leffler function and applications. Filomat. 2020;34(8):2683-2692.

[15] Klimek M, Lupa M. Reflection symmetric formulation of generalized fractional variational calculus. Fract Calc Appl Anal. 2013;16(1):243-261.

[16] Miller KS, Ross B. An introduction to the fractional calculus and fractional differential equations. John Wiley \& Sons: New York, NY, USA; 1993.

[17] Vivas-Cortez M, Nápoles-Valdés JE. Sobre Lyapunov y la Teoría de la Estabilidad. Revista MATUA. 2021;8(1):92-100.

[18] Galeano-Delgado JG, Nápoles-Valdés JE, Pérez-Reyes E, Vivas-Cortez M. The Minkowski Inequality for Generalized Fractional Integrals. Appl Math. 2021;15(1);1-7.

[19] Galeano-Delgado JG, Nápoles-Valdés JE, Pérez-Reyes E. Several integral inequalities for generalized Riemann-Liouville fractional operators. Comm Fac Sc Uni of Ankara S A1 Math and Stat. 2021;70(1):269-278.

[20] Bhanotar SA, Kaabar MKA. Analytical Solutions for the Nonlinear Partial Differential Equations Using the Conformable Triple Laplace Transform Decomposition Method. Int J Differ Equations. 2021;2021:1-18.

[21] Baitiche Z, Derbazi C, Alzabut J, Samei ME, Kaabar MKA, Siri Z. Monotone Iterative Method for $\psi$-Caputo Fractional Differential Equation with Nonlinear Boundary Conditions. Fractal Fract. 2021;5(3):81.

[22] Alzabut J, Selvam A, Dhineshbabu R, Kaabar MKA. The Existence, Uniqueness, and Stability Analysis of the Discrete Fractional ThreePoint Boundary Value Problem for the Elastic Beam Equation. Symmetry. 2021;13(5):789.

[23] Martínez F, Martínez I, Kaabar MKA, Paredes S. New results on complex conformable integral. AIMS Math. 2020;5(6):7695-7710.

[24] Kaabar MKA, Shabibi M, Alzabut J, Etemad S, Sudsutad W, Martínez F, et al. Investigation of the Fractional Strongly Singular Thermostat Model via Fixed Point Techniques. Mathematics. 2021;9(18):2298.

[25] Kilbas AA, Srivastava HM, Trujillo JJ. Theory and applications of fractional differential equations. North-Holland Mathematics Studies. Elsevier Science B.V.: Amsterdam; 2006.

[26] Podlubny I. Fractional differential equations. Academic Press: New York; 1999.

[27] Ma WX, Zhou Y. Lump solutions to nonlinear partial differential equations via Hirota bilinear forms. J Differ Equations. 2018;264(4):2633-2659.

[28] Ma WX. N-soliton solutions and the Hirota conditions in (1+1)-dimensions. Int J Nonlinear Sci Numer Simul. 2021;000010151520200214.

[29] Ma WX. N-soliton solutions and the Hirota conditions in (2+1)-dimensions. Opt Quantum Electron. 2020;52: 511.

[30] Ma WX. N-soliton solution and the Hirota condition of a (2+1)-dimensional combined equation. Math Comput Simul. 2021;190:270279.

[31] Ma WX. N-soliton solution of a combined pKP-BKP equation. J Geom Phys. 2021;165:104191.

[32] Ma WX, Yong X, Lü X. Soliton solutions to the B-type Kadomtsev-Petviashvili equation under general dispersion relations. Wave Motion. 2021;103:102719.

[33] Kaabar MKA, Kaplan M, Siri Z. New Exact Soliton Solutions of the (3+1)-Dimensional Conformable Wazwaz-Benjamin-Bona-Mahony Equation via Two Novel Techniques. J Funct Spaces. 2021;2021:1-13.

[34] Akbulut A, Kaplan M, Kaabar MKA. New conservation laws and exact solutions of the special case of the fifth-order KdV equation. J Ocean Eng Sci. 2021; doi: https://doi.org/10.1016/j.joes.2021.09.010.

[35] Kumar D, Hosseini K, Kaabar MKA, Kaplan M, Salahshour S. On some novel soliton solutions to the generalized SchrödingerBoussinesq equations for the interaction between complex short wave and real long wave envelope. J Ocean Eng Sci. 2021; doi: https://doi.org/10.1016/j.joes.2021.09.008

[36] Darwish MA. On quadratic integral equation of fractional orders. J Math Anal Appl. 2005;311(1):112-119.

[37] Kalvandi V, Samei ME. Mittag-Leffler-Hyers-Ulam-Rassias stability of cubic functional equation. Math and Comp Sci. 2021;1(4):14-21. 
[38] Kalvandi V, Samei ME. New stability results for sum-type fractional q-integro-differential equation. J Adv Math Stud. 2019;12(2):201209.

[39] Samei ME, Hedayati V, Rezapour S. Existence results for a fraction hybrid differential inclusion with Caputo-Hadamard type fractional derivative. Adv Differ Equations. 2019;2019:163.

[40] Fulger D, Scalas E, Germano G. Monte Carlo simulation of uncoupled continuous-time random walks yielding a stochastic solution of the space-time fractional diffusion equation. Phys Rev E. 2008;77(2):021122.

[41] Maamri N, Trigeassou JC. A comparative analysis of two algorithms for the simulation of fractional differential equations. Int J Dyn Control. 2020;8(1):302-311.

[42] Duan B, Zhang Z. A Rational Approximation Scheme for Computing Mittag-Leffler Function with Discrete Elliptic Operator as Input. J Sci Comput. 2021;87(3):1-20.

[43] Özarslan MA, and Fernandez A. On a Five-Parameter Mittag-Leffler Function and the Corresponding Bivariate Fractional Operators. Fractal Fract. 2021;5(2):45.

[44] Mittag-Leffler GM, Sur la nouvelle function of $E_{\alpha}(x)$. C R Acad Sci Paris. 1903;137:554-558.

[45] Wiman A. Uber de fundamental satz in der theorie der funktionen Acta Math. 1905;29:191-201.

[46] Agarwal RP. A propos dune note M. Pierre Humbert, C R Acad Sci Paris. 1953;236:2031-2032.

[47] Humbert P, Agarwal RP. Sur la function de Mittag-Leffler et quelquesunes deses generalizations. Bull Sci Math. 1953;2(77):180-186.

[48] Shukla AK, Prajapati JC. On a generalization of Mittag-Leffler function and its properties. J Math Anal Appl. 2007;336:79-81.

[49] Shukla AK, Prajapati JC. Some remarks on generalized Mittag-Leffler function. Proyecciones. 2009;28(1):27-34.

[50] Salim TO, Faraj O. A generalization of Mittag-Leffler function and integral operator associated with the fractional calculus. J Fractional Calc Appl. 2012;3(5):1-13.

[51] Amit C, Saraswat S. Some remarks on generalized Mittag-Leffler function and fractional operators. IJMMAC. 2014;2(2):131-139.

[52] Desai R, Salehbhal A, Shukla AK. Note on generalized Mittag-Leffler function. SpringerPlus. 2016;5(683):1-8.

[53] Mazhar-ul-Haque M, Holmba TL. A Q function in fractional calculus. J Basic Appl Res Int. 2015;6(4):248-252. 